\title{
DIET: Building Problem Solving Environments for the Grid
}

\author{
Frédéric Desprez \\ LIP Laboratory / GRAAL Project, \\ CNRS, ENS Lyon, INRIA, Univ. Claude Bernard Lyon, France \\ Frederic.Desprez@ens-lyon.fr
}

\begin{abstract}
DIET (Distributed Interactive Engineering Toolbox) is a set of hierarchical components to design Network Enabled Server systems. These systems are built upon servers managed through distributed scheduling agents for a better scalability. Clients ask to these scheduling components to find servers available (using some performance metrics and information about the location of data already on the network). Our target architecture is the grid which is highly heterogeneous and dynamic. Clients, servers, and schedulers are better connected in a dynamic (or peer-to-peer) fashion.

In this keynote talk, we will discuss the different issues to be solved for the efficient deployment of Network Enabled Servers systems on the grid. These issues include the automatic deployment of components, performance evaluation, resource localization, scheduling of requests, and data management. See http://graal.ens-lyon.fr/DIET/ for further information.
\end{abstract}

Biography: Frédéric Desprez is a director of research at INRIA and holds a position at LIP laboratory (ENS Lyon, France). He received is $\mathrm{PhD}$ in C.S. from the Institut National Polytechnique de Grenoble in 1994, his MS in C.S. from the ENS Lyon in 1990, and his habilitation in 2001. His research interests include parallel libraries for scientific computing on parallel distributed memory machines, problem solving environments, and grid computing. See http://graal.ens-lyon.fr/ desprez for further information. 